\title{
The Potential Role of Primary Care Personnel in the Prevention and Screening of Cancer
}

\section{Dobrossy $L^{*}$ and Budai $A$}

Office of Chief Medical Officer, Albert Flórián út 2-4, Budapest, H-1097, Hungary

*Corresponding author: Dobrossy L, Office of Chief Medical Officer, Albert Flórián út 2-4, Budapest, H-1097, Hungary, Tel: +36-536-0-375; E-mail: dobrossy.lajos@oth.antsz.hu

Received date: November 08, 2017, Accepted date: November 10, 2017, Published date: November 14, 2017

Copyright: @ 2017 Döbrőssy L. This is an open-access article distributed under the terms of the Creative Commons Attribution License, which permits unrestricted use, distribution, and reproduction in any medium, provided the original author and source are credited.

\begin{abstract}
The reduction of incidence and mortality of cancer puts a heavy burden on all actors of the health care system. The primary care personnel who see their exclusive role as treating sick people must not be an exception. As a new task, they have to do their part in the prevention aiming at elimination of lifestyle-related risk factors, and in the efforts to detect and treat cancer as early as possible. This paper indicates how they can take part in these efforts, discusses the natural history of cancer, the methods of prevention and screening, and the benefits and potential harms of interventions. Its message is that the efforts which mostly but not exclusively are the tasks of public health could be successful only by the contribution, change of traditional mentality, active and initiative attitude of primary care personnel.
\end{abstract}

Keywords: Cancer screening; Primary care; Health education

\section{Introduction}

With more than 3.7 million new cases and 1.9 million deaths each year, cancer represents the second most important cause of death and morbidity in Europe after cardiovascular diseases, accounting for $25 \%$ of all deaths in 2013, up from 15\% in 1960 . Mortality due to cancer was lowest in Finland, Switzerland and Japan, with rates less than 180 per
100,000 population. Hungary, Slovenia, the Slovak Republic and Denmark bear the highest cancer mortality burden, with rates in excess of 240 per 100,000 population. Both the mortality rates from all causes of death and cancer mortality rates were reported from Hungary (331.1/100,000 for men, and 174/100,000 for women). The epidemiological situation places a heavy burden on all players of the health care system; primary care doctors and nurses are no exceptions (Table 1).

\begin{tabular}{|c|c|c|c|}
\hline Country & Males & Females & Remark (year) \\
\hline Cyprus & 153.1 & 99.0 & 2009 \\
\hline Sweden & 168.1 & 129.5 & 2009 \\
\hline Finland & 174.4 & 114.4 & \\
\hline Malta & 184.3 & 127.7 & \\
\hline Germany & 199.2 & 128.1 & \\
\hline Ireland & 199.9 & 148.0 & \\
\hline United Kingdom & 202.3 & 147.3 & \\
\hline Austria & 203.9 & 125.5 & 2009 \\
\hline Greek & 207.3 & 108.9 & 2009 \\
\hline Luxembourg & 211.7 & 120.0 & \\
\hline Bulgaria & 211.9 & 114.3 & \\
\hline Italy & 212.1 & 122.2 & 2009 \\
\hline Spain & 217.5 & 101.6 & \\
\hline Denmark & 219.4 & 168.2 & 2009 \\
\hline
\end{tabular}


Page 2 of 5

\begin{tabular}{|c|c|c|c|}
\hline Portugal & 220.3 & 108.3 & \\
\hline The Netherlands & 226.9 & 152.0 & \\
\hline Belgium & 227.1 & 129.4 & 2009 \\
\hline France & 228.8 & 116.1 & 2009 \\
\hline Romania & 248.3 & 129.5 & 2009 \\
\hline Czech Republic & 263.2 & 147.5 & \\
\hline Slovenia & 270.3 & 145.8 & \\
\hline Poland & 271.1 & 146.9 & \\
\hline Slovakia & 283.9 & 139.0 & \\
\hline Estonia & 286.0 & 135.7 & 2009 \\
\hline Latvia & 288.0 & 143.3 & 2009 \\
\hline Litvania & 293.2 & 132.5 & 2009 \\
\hline Hungary & 333.1 & 174.9 & \\
\hline EU-27 & 229.8 & 131.7 & \\
\hline
\end{tabular}

Table 1: Cancer mortality in the countries of the European Union 2010.

\section{Natural history of cancer}

The everyday activity of primary care doctors is determined by the traditional mentality that sharply distinguishes between healthy and sick people, disregarding the fact that the healthy status and the sickness are not separated black and withe from each other. Noncommunicable chronic diseases do not emerge overnight. It is a long sequence of events that a tumour goes through before clinically manifesting itself. This process is known as "natural history" of the disease [1]. At the beginning, the normal tissue is exposed to several risk factors, and this might result in the "biological onset of the tumour". This period lends itself for primary prevention that aims to prevent the disease from development. There is another milestone; the appearance of complaints and symptoms, when the patient presents himself/herself to a doctor; this is the beginning of the "clinical phase" of the natural history. Between the two milestones there is a shorter or longer period of time, which may take several years, when there are no complaints or symptoms, but the developing tumour gives a "signal" that can be detected by suitable methods: cells of malignant morphology exfoliate from the cervix uteri, or there is a suspect "shadow" on the mammogram, or occult blood in the stool. This period is named as "preclinical detectable phase" (PCDP). This asymptomatic phase gives way to secondary prevention, i.e. early detection by screening and early treatment by which the advanced or fatal disease can be prevented from happening

\section{Risk factors}

The cause of diseases might be any factor, which is necessary and sufficient to cause a certain disease (Koch-postulate). The definition is fully compliant to communicable diseases, but does not apply to the non-communicable chronic disease, such as cancer. The causes of the majority of cancers are not sufficiently understood. Epidemiological evidence has suggested, however, that many different factors are not necessarily causal agents but associated, individually or in combination, with an increased likelihood, or risk of the occurrence of certain diseases. These factors that might have a role to play in development of diseases are widely denoted as determinants, predisposing or risk factors. Our perception on risk factors has gone through substantial changes over the last decades. A Working brought together by the World Health Organization in 1964 was the first to announce that the most common cancers are caused by extrinsic or environmental risk factors (such as air pollution, water pollution, food additives, occupational and industrial factors, etc.), therefore are potentially avoidable, or preventable [2]. Recently, the role of the "personal environment" or "lifestyle" has been increasingly emphasized (Figure 1) [3].

\section{Natural history of disease development}

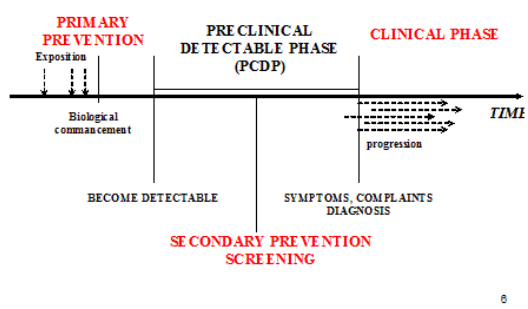

Figure 1: Natural history of disease development. 


\section{The lifestyle concept}

It goes back to Marc Leland, the former minister of health, Canada, who suggested the "lifestyle concept" to introduce into the glossary of the health field [4]. In 1974, Lalond published a working document entitled "A new perspective on health of Canadians". Previously, it was considered that, theoretically, the function of health field has had two pillars: biology (including both an individual's genetic make-up, family history, process of maturation and aging etc., and the accumulated biomedical knowledge) and health care organization (human and physical resources that affect access to medical care, including medical practice, nursing, hospitals, nursing homes, medical drugs, public health services, paramedic services, dental treatment and other health services). Lalond suggested two additional pillars to take into account: environment (both physical and social, which surrounds individuals and shape behaviour, positive or negative); and finally, the lifestyle. In this context, the lifestyle concept-not as sociological one, but in terms of disease development and prevention-has been defined as "aggregate of personal decisions that can contribute to, or cause illness and health, over which the individual has control", or "behaviour which includes individual responses to individual stimuli or external conditions, as demonstrated through decisions by individuals that effect their risks and their health status." The lifestyle-related risk factors are as follows: tobacco smoking, poor diet, alcohol consumption, overweight and obesity, physical inactivity, increased exposure to sunlight (UV-B), some reproductive factors. The exposure to lifestyle-related risk factors and development of the majority of most common cancers has been proven by epidemiological data [5].

The Lalond Report is considered the "first modern government document in the Western world to acknowledge that our emphasis upon a biomedical health care system is wrong, and that we need to look beyond the traditional health care (sick care) system if we wish to improve the health of the public" [6]. It has had a worldwide effect in making governments and health professionals realize that the promotion of health in the future depends more on the pattern of living adopted by the individuals than on technical or allied procedures.

It has been recognized that an excessive emphasis on lifestyle could lead to a "blame the victim" mentality. Smoking, for example, was not merely a matter of personal choice but also a function of one's social environment. As a result, physical and social environments were differentiated, with growing emphasis placed on the latter [7]. It is important to remember that the lifestyle-related risk factors are avoidable, thus the majority of lifestyle-related cancers are-in theorypreventable.

It is important to note that the risk factors are common for most non-communicable diseases. This means that the prevention of cardiovascular diseases and cancer can be separated only by didactical considerations: when the primary care personnel take pains to prevent any life-style related disease, they serve the prevention of both cardiovascular disease and cancer simultaneously.

\section{Primary prevention}

The ultimate aim of disease prevention is to avoid or at least to reduce by proper intervention the exposure of individuals and the community to known avoidable risk factors, thereby preventing the onset of the disease. Preventive strategy can focus on the population as a whole through government regulations or health education of the public (population strategy), or on people identified as being at high risk of certain disease (high risk strategy) [8]. For obvious reasons, the high-risk strategy has come to dominate the medical approach to prevention. This particularly applies to primary physicians and nurses, as health care workers who are role models, opinion formers and leaders in all matters that influence health.

\section{Secondary prevention: Screening}

Another aim of prevention is to favourably modify the course of disease development by detection and treatment in an early, preclinical stage, when the disease is more responsive to curative treatment, thereby preventing clinically manifest, advanced disease (secondary prevention). The means to achieving this aim is screening, which is defined as regular examination by suitable methods of people having no symptoms or complaints, with the aim of detecting the target disease earlier than it would have been detected without screening, or to rule out with great probability of the existence of the disease. Such targets for screening might be an early, non-invasive cancer, or precancerous lesion, which is a ground for the development of cancer.

\section{Criteria for screening}

For screening, the following criteria must be met [9]:

- the target disease should be of public health importance;

- the natural history well known with a long preclinical detectable phase;

- suitable method is available for detection of hidden, asymptomatic lesion; the method is accepted for the target population;

- the treatment of the detected lesion is available, and has a greater chance for cure.

\section{Opportunistic and/or organized screening}

In the health care system, two kinds of screening practice can be distinguished [10]. Opportunistic screening is performed as part of the medical practice, linked to a physician-patient encounter for any other reason. The examination can be initiated by the physician, motivated by the "oncological alertness"; or by the patient herself/himself asking for it, seeking "peace of mind" that he/she is in "good health". As a result of the health education of the public at large, more-and-more patients go to primary physicians requesting some sort of screening test.

Organized screening, initiated and financed by the provider (health care system) in order to make participation in the screening optimal. As such, it is an activity of public health. The management of the screening programme sets up a list of persons eligible for screening, and sends them a personal invitation letter indicating the site and time of the examination. If the test result is negative, the patient is recalled for repeated screening in two-three years' time. In case of a nonnegative (positive) result, the patient is referred to specialist for confirmatory or diagnostics and, if necessary, for treatment.

It is an important "rule of game" that organized screening can be initiated only in those screening modalities when, attributable to the screening activities, the mortality from the target disease is substantially reduced in the target population. For the time being, there are not more than three screening modalities of epidemiologically proves effectiveness:

- Cytology-based cervical screening in women between 25 and 64 years of age every three years; 
- Biannual breast screening by mammography in women between 45 and 65 years of age; and

- Biannual colorectal screening of males and females between 50 and 70 years of age by faecal occult blood test (FIT).

According to the "rules of the game", no screening modality should be offered on a large scale without conclusive evidence of its effectiveness in reducing mortality from the target disease, or improving quality of life by permitting less aggressive treatment. Those screening modalities for which the epidemiological evidence of effectiveness still lacking, the call-and recall screening system must not be applied. These modalities can be encouraged to be applied on the basis of medical judgement, clinical indications, or taking advantage of any forms of medical-patient encounter. Such modalities are as follows: prostate screening by PSA [11], screening for oral cancer by palpation and inspection [12], or selective screening for lung cancer by low-dose helical CT [13].

\section{Role of primary care in prevention and early detection of cancer}

The primary care personnel, both physicians and nurses, have unlimited opportunities in both primary prevention and screening. In their practice, distribution of the entire population by age and gender, social, economic and lifestyle indicators are being reflected. They are dealing with not only sick people, but several other target groups relevant to prevention and early detection, such as healthy persons conducting healthy or health-damaging lifestyle, apparently healthy persons with still asymptomatic preclinical disease.

This means that their everyday work present hundreds of opportunities for a large-scale, people-oriented preventive activity. Such activities are: individual risk assessment, health education, personal counselling, and promotion of attendance in organised screening, in particular.

\section{Individual risk assessment}

The primary physician has plenty of opportunities to reflect upon whether his/her patient has had self-damaging, health-damaging habits by asking questions about smoking habits, hazardous alcohol consumption or unhealthy diet, measuring body-mass index (BMI), and several other ways. The risk factors need to be evaluated in conjunction with each other, as they might have a role to play in combination.

\section{Health education and counselling}

The aim of health education is to provide information to the patient about lifestyle-related health-damaging factors, and the relationship between risk factors and certain diseases. The familiarization of risk factors might be followed by the personal counselling about what can be done to diminish the risk of health damaging.

For instance, measuring the weight and height gives an opportunity to talk about the balance on diet (calorie intake) and physical activity (decrease of calorie intake). This could make sure that the counselling would be not only professionally correct but personalized. In any communication with individuals, the team members have to deliver advice on lifestyle in a way that the recipients feel it is not intrusive and authoritative but personally relevant.

\section{Promotion of organized population screening}

In most countries, the duties of primary care personnel in organised population screening are regulated by legislation, but the regulations are less than satisfying when it comes to their opportunities in screening for cancer. No doubt, the primary care personnel are the "gatekeepers" of population screening. Their influence on the acceptance of the population of the offered screening is obvious. Their role is more than to raise attention and to refer them to screening, but to motivate and stimulate those invited for screening examination to participate in the examination. The primary physicians-having their patients' confidence-are most able to obtain credit for the screening, and persuade them to participate. Furthermore, they are expected to register the test results, and to refer those with a non-negative (positive) test result to the respective diagnostic department for confirmatory examination.

The primary care personnel can contribute to the performance of the various screening modalities in different ways. As to cervical screening, it is the duty of the contracted cytology laboratories that analyse the Pap-smears or put the HPV test through. In some countries primary care physician or his/her assistants, primary health nurses, midwifes and, exceptionally, gynaecologists take the smear. In mammography screening, the examination is generally the task of radiologists; the primary care personnel have but to stimulate the invited patients to attend the examination. Screening for colorectal screening is the only modality where the active participation of the primary care personnel is needed, as the general recommendation is to put the tools and instructions for the stool collection installed into the primary care office, and they are to distribute to those to be screened, and to refer those with positive test result to colonoscopy.

\section{Ethical responsibility}

Preventive practices carry considerable ethical responsibility to do more good than harm, or no harm at all. The primary care team that provides preventive services to people who are healthy-or believe they are healthy-have an ethical obligation to maximize the benefit of the intervention and to minimize its potentially harmful effects. While people may consider public information, education or advice on lifestyle issues as an uninvited intrusion of medicine into their personal lives, it alerts them to how dangerous they can be to their own health [14]. As a result, people's sense of wellbeing declines. Encouraged to look for warning signs and danger signals in their bodies, healthy people may suffer mental unrest, fear and anxiety about having some dreadful disease sometime in the remote future. On the contrary, people are supposed to consider all information and advice as a positive contribution to their health, because the intention is to benefit them by promoting and protecting their health.

The primary care team must respect a patient's right to be fully informed about the possible limitations of screening tests and hazards of the procedures. They need to provide all information necessary to make informed decision about whether to accept the offered measure [15].

\section{Psychological side-effects of screening}

The primary care personnel need to handle the avoidable psychological side effects. The invitation to screening is an unwanted intrusion into the private sphere of an individual. It forces the patient to decide without knowing the consequences of accepting or rejecting an invitation. They know nothing of what is going to happen to them, 
Page 5 of 5

and only a little of why. They are most likely to accept the invitation so that later they would not regret the rejection of it (anticipated decision regret) [16]. Often the mere mention of the suspicion of the illness adheres to the patient a "label of the illness", adversely affecting his/her self-image ("medicalization of risk") [17]. Concern may be caused by a non-negative test result whose psychological effect is not determined by the pathological abnormality behind it, but by the subjective living of the individual; this is particularly true in those cases when the screening is "suspect", and only the subsequent diagnostic test results in "acquittal judgment". In the waiting period, uncertainty may be a nightmare for the patient. The primary care personnel have to provide psychological support to the patients in case of "bad news", because with a positive test result the fear of possible consequences can overwhelm the patients.

Furthermore, each test has inherent limitations, and has proven psychological adverse effects, such as anxiety and fear due to the uncertainties before the test is performed, or while waiting for the test results, and distress caused by abnormal, particularly false-positive test results and the process of verification. For instance, people may be labelled as having "precancer" when mild or moderate dysplasia is found [18]. It is the duty of primary care personnel to provide psychological support to their patients through these difficult periods.

\section{Conclusion}

According to the traditional conception, practicing physicians see their role as healing sick people. They consider the promotion and protection of health and prevention of diseases as the task of public health, exclusively. The prevention, early detection and screening of diseases require a change in traditional medical behaviour: an active, in initiative, exploratory one, rather than the usual which is called by an early author as "shopkeeper attitude", quoted: "Like it or not, the working tradition from which general practice stem is the local sick shop, wherein the doctor, thinly disguised as a scientific gentleman, remain a shopkeeper. His contact with the population at risk remains a shopkeeper" [19].

The potential of primary and secondary prevention in the primary care is underutilized. This could be resulted from insufficient knowledge of primary care personnel. They are not adequately motivated. They refer to lack of time and being overloaded, lack of financial incentives. They doubt the benefit of preventive efforts. And so on, one could continue.

The improvement of a currently unfavourable health status of the population around the world requires the integration of prevention, early detection and screening of malignant tumours into the daily activities of primary care personnel.

\section{References}

1. Miller AB (1992) The natural history of cervical cancer and implications for screening policy. In: Miller AB (eds) Cervical screening programmes. WHO Geneva 3: 115-123.

2. World Health Organization (1964) Prevention of cancer. WHO Geneva.

3. Doll R, Peto R (1981) The causes of cancer. J Natl Cancer Inst 66: 1191-1308.

4. http://www.phac-aspc.gc.ca/ph-sp/pdf/perspect-eng.pdf

5. Wiederpass E (2010) Lifestyle and cancer risk. J Prev Med Public Health 43: 459-471.

6. Hanock T (1985) Beyond health care: From public health policy to healthy public policy. Can J Public Health 76: 9-11.

7. Richards H, Reid M, Watt G (2003) Victim-blaming revisited: A qualitative study of beliefs about illness causation, and responses to chest pain. Fam Pract 20: 711-716.

8. Rose G (1992) The strategy of preventive medicine. Oxford University Press.

9. http://apps.who.int/iris/bitstream/10665/37650/17/WHO_PHP_34.pdf

10. Hakama M, Miller AB, Day NE (1986) Screening for cancer of uterin cervix. Lyon, pp. 1-305.

11. Moyer VA, U.S. Preventive Services Task Force (2012). Screening for prostate cancer: U.S. preventive services task force recommendation statement. Ann Intern Med. 157: 120-34.

12. Olson CM, Burda BU, Beil T, Whitlock EP (2013) Screening for oral cancer. A targeted evidence update for the U.S. preventive services task force. Evidence Syntheses 102.

13. Kramer BS, Berg CHD, Aberle DR, Prorok PC (2011) Lung cancer screening with low-dose helical CT: Results from the National Lung Screening Trial (NLST). J Med Screen 18: 109-111.

14. Crawford R (1977) You are dangerous to your health: The ideology and politics of victim blaming. Int J Health Serv 7: 663-680.

15. Rimer BK, Briss PA, Zeller PK, Chan EC, Woolf SH (2004) Informed decision making: What is its role in cancer screening? Cancer 101: 1214-1228.

16. Tymstra $\mathrm{T}$ (1989) The imperative character of medical technology and the meaning of "Anticipated Decision Regret". Int J Technol Assess Health Care 5: 207-213.

17. Gotzsche PC (2002) Commentary: Medicalization of risk factors. Brit Med J 324: 890-891.

18. Brawley OW, Kramer BS (2005) Cancer screening in theory and in practice. J Clin Oncol 23: 293-300.

19. Hart JT (1977) A new kind of doctor. J Royal Soc Med 74: 871-883. 